\title{
AVILÉS, ENTRE EL DECLIVE Y LA REVITALIZACIÓN: ¿EN LA GÉNESIS DE UN NUEVO MODELO DE DESARROLLO?'
}

\author{
Simón Sánchez Moral \\ Departamento de Análisis Geográfico Regional y Geografía Física, Universidad Complutense de Madrid \\ simon.sanchez@ghis.ucm.es \\ Ricardo Méndez \\ Consejo Superior de Investigaciones Científicas \\ ricardo.mendez@cchs.csic.es \\ José Prada Trigo \\ Consejo Superior de Investigaciones Científicas \\ pradatrigo@gmail.com
}

\section{RESUMEN}

Tras tres décadas de retroceso demográfico y severos ajustes sobre el empleo provocados por la reconversión industrial iniciada en los años ochenta, la ciudad de Avilés ofrece síntomas de recuperación. El artículo profundiza en las claves explicativas tanto del declive urbano como del resurgimiento, destacando la aparición de una nueva gobernanza urbana. Esta habría permitido la puesta en marcha de estrategias de revitalización económica y regeneración urbana y, sobre todo, alcanzar el consenso en torno a un nuevo proyecto de ciudad, que el trabajo examina a la luz de algunos proyectos estructurantes contenidos en el nuevo Plan General (2007).

Palabras clave: Desindustrialización, declive y resurgimiento urbanos, gobernanza.

Fecha de recepción: marzo 2011.

Fecha de aceptación: julio 2012.

1 Este trabajo forma parte de la investigación «Cities Regrowing Smaller - Fostering Knowledge on Regeneration Strategies in Shrinking Cities across Europe», financiada por la Comisión Europea a través del Programa COST (TU0803) (2009-2013).

Simón Sánchez Moral es investigador del subprograma Ramón y Cajal del Ministerio de Ciencia e Innovación. 


\section{ABSTRACT}

After three decades of decline and drastic adjustments on employment caused by the industrial restructuring that began in the eighties, the city of Aviles offers signs of recovery. The article delves into the key explanations for the decline both urban and resurgence, highlighting the emergence of a new urban governance. This has allowed the implementation of strategies for economic revitalization and urban regeneration and, above all, reach consensus around a new city project, the paper examines in the light of some structural projects contained in the new Master Plan (2007).

Key words: Deindustrialization, urban decline and resurgence, governance.

\section{INTRODUCCIÓN}

Dentro de la diversidad de trayectorias urbanas que durante las últimas décadas se han integrado bajo la denominación genérica de ciudades en declive (siendo la pérdida continuada de población sólo un primer indicador del fenómeno) las existentes en España corresponden en su mayor parte a ciudades mineras e industriales especializadas en sectores intensivos en recursos naturales y mano de obra, localizadas sobre todo en el Arco Atlántico, que padecieron la crisis del fordismo hace al menos tres décadas. Sus impactos económicos y laborales, pero también sociales y ambientales, provocaron un declive que tan sólo algunas parecen haber superado, en contraste con la atonía y falta de expectativas que aún aquejan a otras (Sánchez Moral, Prada y Méndez, 2009).

En ese contexto, Avilés es una ciudad de tamaño medio (84.242 habitantes, que llegan a 124.100 en su área urbana), situada en el litoral de Asturias, la región española más afectada por esos procesos. La crisis de su cluster siderometalúrgico, dominado por una gran empresa pública, Empresa Nacional Siderúrgica (Ensidesa) instalada a mediados del siglo XX y su posterior reconversión generaron una fuerte reducción de empleos, el retroceso demográfico e importantes tensiones sociales. La tradicional dependencia de decisiones externas, la escasez de iniciativas empresariales y el agotamiento del modelo de crecimiento impuesto durante décadas provocaron también la ausencia de perspectivas de futuro y un notable pesimismo en la sociedad local, componentes intangibles pero no banales de los procesos de declive urbano.

Sólo desde la última década del pasado siglo comenzó a definirse un nuevo proyecto de ciudad, a partir del efecto provocado por determinadas políticas de promoción de los gobiernos central y regional o de la Comisión Europea. Pero, sobre todo, a partir de una serie de iniciativas, que pueden calificarse de innovadoras, promovidas por los actores locales, tanto públicos como privados, que han puesto en práctica nuevas formas de gobernanza destinadas a potenciar algunos ejes estratégicos para el desarrollo local. Por una parte, la renovación de su base industrial mediante el redimensionamiento y privatización de la siderurgia, el apoyo a proyectos de innovación en las pequeñas empresas y la ampliación del puerto, elemento central del desarrollo urbano a lo largo de su historia. Por otra, la apuesta por una economía local más diversificada que valora el turismo y la industria cultural como sectores estra- 
tégicos complementarios y tiene su reflejo en algunos proyectos estrella para posicionar internacionalmente a la ciudad. Finalmente, una mejora del entorno urbano y la calidad de vida, con actuaciones destacadas como la restauración ambiental y paisajística de la ría, la rehabilitación del centro histórico o la mejora de equipamientos en los barrios entre otras.

El objetivo del texto es caracterizar la transición de Avilés desde una situación de declive a otra que puede calificarse como de resurgimiento, comenzando por un breve contexto teórico que recuerda los argumentos principales que han guiado la investigación, dentro de los planteamientos generales que propone el concepto de shrinking city («ciudad menguante») (Fol y Cunningham-Sabot, 2010). A partir de aquí, se hace una aproximación descriptiva a los principales indicadores de los cambios producidos, se destaca la influencia ejercida por los actores locales o externos y por la densificación de las redes socioeconómicas en la construcción de un nuevo marco institucional, para continuar con una selección y análisis de las principales acciones emprendidas y finalizar con unas conclusiones en que también se apuntan algunas debilidades aún no resueltas.

Para llevar a cabo la investigación se elaboró una amplia base de datos estadísticos mediante la utilización de diversas fuentes, junto con numerosos documentos de planeamiento, estudios publicados en los últimos años y noticias recogidas de la prensa local o regional. Pero la fuente principal de información, que ha hecho posible interpretar las claves de los procesos analizados, ha sido el trabajo directo realizado en la ciudad de Avilés y las entrevistas semiestructuradas a un total de 17 actores locales representativos de los diferentes sectores de la sociedad y las instituciones locales, a los que queremos hacer presente nuestro agradecimiento.

\section{FACTORES DE DECLIVE Y REVITALIZACIÓN EN CIUDADES DE TRADICIÓN INDUSTRIAL}

La evolución de las ciudades está marcada por etapas en las que tanto su población como sus actividades y funciones, sus relaciones con el exterior y su dinamismo experimentan importantes cambios que afectan de forma directa a la vida de sus ciudadanos. En ese proceso son numerosas las que se han enfrentado, en el pasado o en el presente, a una crisis que cuestionó su desarrollo y, en algunos casos, hasta su pervivencia. En ocasiones, esa crisis se debió a catástrofes puntuales de origen natural o humano, que tuvieron efectos destructivos, pero resultan bastante más frecuentes los procesos asociados a transformaciones tecnológicas, económicas o políticas (cambio de la base energética, nueva organización productiva, liberalización de mercados, desaparición de sistemas políticos, etc.), que son el origen de procesos de declive de larga duración.

Hace ya más de tres décadas, la crisis del sistema de producción fordista, la revolución de las tecnologías de información y comunicación, así como todo un conjunto de cambios organizativos, sociolaborales y políticos vinculados al proceso de globalización cuestionaron el futuro de determinadas regiones europeas que habían basado su dinamismo en un modelo que entonces se agotaba. Se trató, sobre todo, de territorios monoespecializados en actividades extractivas dependientes de recursos minerales o en industrias intensivas en el uso de energía, materias primas y mano de obra, poco competitivas ya frente a las de países emergentes con menores costes. Esas regiones de antigua industrialización padecieron unos procesos a menudo traumáticos, así como el desigual impacto de diferentes políticas 
de reconversión y revitalización aprobadas por la Comisión Europea y los gobiernos de sus respectivos países, que provocaron su retroceso relativo por comparación con otras con una base socioeconómica más diversificada, mayor presencia de trabajadores cualificados y actividades expansivas (Ballance y Sinclair, 1983; Fothergill et al., 1988; Landabaso y Díez, 1989).

En algunas interpretaciones teóricas, esos fenómenos de desindustrialización se asociaron con una tendencia general hacia la desurbanización o contraurbanización en los países más avanzados, identificándose incluso distintas fases dentro de un ciclo de vida urbano que relacionaba la terciarización económica, la suburbanización y la pérdida de dinamismo de los centros urbanos (Van den Berg et al., 1982; Cheshire y Hay, 1989). No obstante, la recuperación del crecimiento en las décadas siguientes puso de manifiesto que el declive se concentraba en determinadas ciudades, aquejadas por una acumulación de desventajas comparativas que provocaban una pérdida de capacidad para evitar el cierre o deslocalización de una parte de sus empresas, atraer nuevas inversiones y mantener así a su población ante la falta de expectativas (Rhodes, 1986).

En fechas más reciente, la desaparición de las economías de planificación centralizada, con la crisis de sectores y empresas incapaces de resistir la rápida transición hacia una economía de mercado, la destrucción masiva de empleos y su especial concentración en determinadas regiones y ciudades industriales, portuarias y mineras, revitalizó una temática del declive regional y urbano ahora centrada, sobre todo, en la otra margen del continente (Birch, MacKinnon y Cumbers, 2010). Al mismo tiempo, se acuñó el concepto de shrinking city o ciudad menguante para aludir a este tipo de procesos (Fol y Cunningham-Sabot, 2010), bastante significativos en el conjunto de las áreas urbanas europeas durante la última década (Turok y Mykhnenko, 2007).

En la explicación de la evolución negativa que siguen mostrando determinadas ciudades, más allá de los procesos estructurales desencadenantes suele prestarse especial atención a los bloqueos endógenos en forma de determinadas estructuras heredadas (económicas, sociolaborales, institucionales...) que limitan su capacidad de adaptación, una de las manifestaciones del principio de path dependence (Martin y Sunley, 2006; Simmie y Carpenter, 2008) que pone de manifiesto el carácter acumulativo de las decisiones, las inercias derivadas y su influencia sobre trayectorias locales que se transforman a menudo de forma lenta.

No obstante, entre las ciudades que se han enfrentado en las últimas décadas a procesos de declive agudo existe un creciente número de evidencias (Power et al., 2010) que demuestran la capacidad de algunas de ellas para revertir esa tendencia y recuperar tasas de crecimiento positivas que ha permitido hablar de un verdadero resurgimiento (Turok y Mykhnenko, 2008), lo que las convierte en objeto de creciente atención en la investigación actual sobre desarrollo urbano. Los intentos de comprender las claves que subyacen a esa desigual trayectoria de ciudades enfrentadas en su pasado reciente a dificultades similares se corresponden con tres perspectivas de especial interés para este tipo de objetivo.

Están, por un lado, los enfoques relacionales que entienden el territorio como una construcción social tejida por múltiples redes de actores con características diversas, pero que en contextos institucionales determinados pueden establecer relaciones de cooperación para negociar estrategias compartidas de desarrollo (Sunley, 2008). Un segundo grupo lo forman aquellos que han centrado su atención en la capacidad de ciertas ciudades para construir 
ambientes innovadores en lo económico y lo social, en busca de nuevas respuestas frente a los retos actuales (Méndez, 2002; Camagni y Maillat eds., 2006). En la actualidad emerge la metáfora de la resiliencia urbana, definida como «la capacidad de ciertas economías locales para transformarse frente a los shocks tecnológicos que socavan su base económica» (Polèse, 2010: 8), o como «un concepto que intenta capturar la distinta y desigual capacidad de los lugares para reaccionar, responder y adaptarse a la incertidumbre, la volatilidad y el cambio acelerado» (Pike, Dawley y Tomaney, 2010: 59), proponiéndose también claves explicativas próximas a las anteriores.

Sin abordar ahora una revisión pormenorizada de esa abundante bibliografía, esos distintos enfoques comparten la idea de que la diferente evolución reciente de las ciudades en declive no puede explicarse tan sólo por el impacto de los procesos estructurales, su accesibilidad a los centros económicos o la especialización sectorial heredada, siendo necesario acudir a la existencia de factores locales para explicar en qué parecen cimentarse los procesos de revitalización de mayor éxito.

El primero de ellos es la decisión y el esfuerzo de diferentes actores urbanos, tanto del sector público como del ámbito empresarial o la sociedad civil, por promover estrategias de respuesta a la globalización liberal desde el interior de la propia ciudad, cualquiera que sea su tamaño o su localización en el territorio. Eso conlleva un eficaz aprovechamiento de sus recursos específicos, construidos en el tiempo, entre los que el capital humano, el cultural, el social y el intelectual cobran creciente importancia en relación a los de carácter material. En ese contexto, la capacidad de liderazgo mostrada por los gobiernos locales, tanto para promover iniciativas como para gestionarlas y servir de catalizador que aglutine a otros actores con intereses contrapuestos en torno a un proyecto de ciudad se considera estratégica (Méndez ed., 2010).

Otro factor fundamental es la capacidad de las empresas instaladas en el territorio para constituir redes de cooperación, formales o informales pero relativamente estables, para transmitir a través de ellas información y conocimiento, en particular de carácter tácito, compartiendo proyectos y generando diversos tipos de externalidades. Por último, resulta imprescindible hablar del marco institucional y la existencia de redes sociales de cooperación, de carácter multiescalar, entre actores públicos y privados que a su proximidad espacial unen la derivada de códigos y lenguajes comunes, capaces de generar una más eficaz gobernanza territorial (Caravaca y González, 2009; Salom y Albertos eds., 2009). Ésta se basa en la existencia de un poder ejercido y negociado a través de un reparto de responsabilidades, eliminando modelos rígidos de gobierno en favor del interés colectivo, así como en el establecimiento de valores, normas y organizaciones favorables a la iniciativa, la colaboración o la concertación. La existencia de un marco institucional adecuado puede actuar así como agente catalizador del desarrollo, al poner en relación a los diferentes actores locales y promover relaciones de confianza, necesarias para abordar formas de gobernanza más participativas, que acumulan recursos y dotan de mayor legitimidad a las decisiones colectivas (Kooiman, 2003, Pascual y Godàs eds., 2010), lo que «permite acumular un capital social, intelectual y político que se convierte en un recurso» (Healey, 1997: 311).

Finalmente, en un mundo cada vez más interdependiente, tejido por múltiples redes de flujos que conectan lugares de formas y en escalas muy distintas, resulta también importante conseguir una buena inserción de las ciudades en su conexión con el exterior. Lograr una 
mayor presencia internacional, una mejor coordinación con las instituciones de ámbito estatal y regional, o una participación en redes de ciudades para colaborar en proyectos comunes, intercambiar experiencias de buenas y malas prácticas o propiciar una mayor capacidad de interlocución en la defensa de determinados intereses pueden ser estrategias que también parecen propiciar la construcción de ciudades resilientes o en proceso de regeneración. El caso de estudio elegido resulta de especial interés para contrastar de forma crítica este tipo de ideas, poner de manifiesto la importancia de la reflexividad en la definición de una estrategia local de desarrollo y sugerir posibles líneas de acción.

\section{LA DESINDUSTRIALIZACIÓN EN EL ORIGEN DEL DECLIVE URBANO DE AVILÉS}

Cuando hacia 1950 el Estado español decide instalar una planta siderúrgica integral en la ciudad, Avilés cuenta con poco más de 21.000 habitantes. En los cinco años previos a la inauguración de Ensidesa se alcanzaron ya los 34.251 habitantes, ante la llegada masiva de trabajadores a una ciudad cuya dinámica demográfica había estado marcada hasta ese momento por el estricto crecimiento vegetativo y, en todo caso, por la emigración, especialmente durante el siglo diecinueve y la primera mitad del veinte. Convertido en uno de los mayores centros de inmigración en masa del país hasta comienzos de los años setenta, momento en que parece que el flujo migratorio cesó definitivamente, Avilés alcanzó los 85.299 habitantes en 1975. El crecimiento explosivo de la población supuso no sólo un choque demográfico enorme, sino también social y cultural; la ciudad en su totalidad es considerada como un «gigantesco experimento controlado por el aparato del Régimen franquista, orgulloso de su realización» (De La Madrid, 1999: 259).

Hacia mediados de la década alcanzaba su máximo desarrollo el cluster siderometalúrgico de la ciudad-comarca de Avilés armado en torno a Ensidesa, que en 1968 ya se había hecho con los activos de otra empresa pública previamente instalada en la margen izquierda de la ría, Siderurgia Asturiana (Siasa). Tras la absorción en 1973 de la Unión de las Siderurgias Asturianas (Uninsa), el gigante público que en 1960 contaba con 7.502 trabajadores, alcanzó los 27.244. Ello significó, además, la consolidación del cluster a escala subregional, al incluir otras grandes empresas públicas, como la Empresa Nacional de Aluminio (Endasa) o Asturiana de Zinc (AZSA), además de un tejido auxiliar de PYMEs, reforzándose así la primacía del corredor siderúrgico entre Avilés y la vecina ciudad de Gijón frente al foco del interior de Asturias, que entró en una situación de profundo declive (Prada, 2011: 60). A todo ello hay que sumar la presencia de otras muchas empresas industriales, también en el entorno del puerto y la ría, destacando Enfersa, que creó una planta química asociada a la siderurgia, y Cristalería Española (CESA).

A modo de ejemplo, el sector siderometalúrgico de la comarca suponía en ese año 1975 el 42,2\% de la población activa de la comarca; Ensidesa producía el 30\% del acero español, Endasa el $43 \%$ del aluminio y AZSA el $82 \%$ del zinc. El impacto en el territorio de este acelerado proceso de industrialización - y, más aún, de la posterior desindustrialización que sufrirá Avilés - será muy grande, dejando tras de sí una herencia que en determinados aspectos es aún hoy visible.

La falta de planificación que acompañó al espectacular crecimiento demográfico de la villa desde la década de los 50 dio lugar a intensos desequilibrios territoriales. De esta forma, 
a un centro urbano con escaso crecimiento hasta ese momento se le añadieron poblados obreros, núcleos periféricos e incluso barrios extramunicipales, de tal forma que se generó un hábitat obrero disperso, inconexo, con un alto grado de segregación social en origen y un acusado carácter de provisionalidad, visible en los déficit en equipamientos y servicios (Benito, 1992: 255-256). Algunos de estos espacios contaban, además, con sus propios hospitales, escuelas, economatos y quedaron al margen del planeamiento urbano y de la propia vida del centro de la ciudad, donde mayoritariamente residía la población autóctona. En esos primeros momentos se evidenciaba igualmente que la gran industria, «dueña y señora del territorio», se implantó con gran despilfarro de suelo, acumulando nuevas instalaciones sin apenas reciclado de las anteriores y haciendo gala de una visión unidimensional y pretendidamente funcional, que no tenía en cuenta posibles incidencias negativas (Leira y Calvo, 2003). Una de las más evidentes fue el fuerte deterioro ambiental que habría de sufrir Avilés, que a comienzos de los ochenta se consideró la ciudad española más contaminada y únicamente superada a nivel europeo por la ciudad polaca de Katowice.

A partir de ese momento culminante, la crisis mundial de los años setenta, que trajo consigo el aumento de los costes empresariales, la contracción de la demanda y una reestructuración de las grandes corporaciones de la siderurgia integral europea (Usinor-Sacilor, British Steel o Cockerill Sambre), unida a la política de reconversión impulsada desde el Estado español en la década de los ochenta y a la apertura del mercado siderúrgico tras la integración en la Comunidad Europea, situaron a Avilés ante el escenario de la desindustrialización. Los déficits tecnológicos y organizativos que lastraban la productividad de Ensidesa acentuaron su vulnerabilidad frente a ese nuevo contexto, en especial tras la absorción, en 1973, de Uninsa, empresa deficitaria que restó aún más productividad a Ensidesa.

En una situación que se repitió en muchas otras regiones y ciudades europeas de antigua industrialización, Avilés tuvo que hacer frente a un nuevo contexto productivo, marcado por las nuevas formas flexibles de organización de la actividad. De un lado se constató el aumento de la competencia internacional en sectores maduros intensivos en recursos naturales y trabajo (entre ellos un sector siderúrgico con problemas de comercialización por el escaso nivel de transformación de sus productos y el elevado precio de algunos de ellos), incapaces de satisfacer las crecientes demandas de innovación. Al mismo tiempo, la reducción de los costes de transporte y el potencial difusor de las TICs reforzaron la tendencia a la segmentación espacial de las actividades según ventajas comparativas y su deslocalización, como respuesta a la creciente competencia en costes de este tipo de sectores. A todo ello se sumaron unas deseconomías de aglomeración crecientes y fuertes externalidades negativas en ciudades de antigua industrialización como esta, junto con las mayores restricciones urbanísticas y ambientales, lo que en conjunto penalizaba la instalación de nuevas iniciativas empresariales. Ante este panorama, la fuerte especialización siderúrgica heredada por Avilés, el peso de la gran empresa pública y la ausencia de actividades urbanas capaces de compensar la pérdida de dinamismo económico condicionaron su capacidad de adaptación ante las nuevas circunstancias (Benito, 2004).

Con retraso respecto de las acciones emprendidas en Europa, las autoridades españolas acometerían un primer Plan de reconversión de la siderúrgica integral (1984-1990), centrado en las empresas Ensidesa y Altos Hornos de Vizcaya, o lo que es lo mismo, Avilés y 
Bilbao, este segundo caso convertido en ejemplo paradigmático de la reconversión industrial y de las estrategias de revitalización en España (Esteban, 2000; González, 2006) y desde luego en primera referencia para la actual estrategia de desarrollo urbano de Avilés analizada en este trabajo. Una segunda fase más dura, identificada con el Plan de competitividad (1991-1997), significó la desaparición de Ensidesa en 1995 que, al igual que el resto de siderurgias españolas, quedaba englobada en la Corporación Siderúrgica Integral (CSI), participada al $50 \%$ por el capital público. Tras un proceso de saneamiento y redimensionamiento, la empresa fue vendida en 1997 a Aceralia, de capital luxemburgués, que en 2002 se integraba en la multinacional Arcelor-Mittal.

Con el cierre en la primavera de 1998 del último de los cuatros altos hornos con que llegó a contar Avilés en la década de los setenta culminaba el desmantelamiento de su cabecera siderúrgica, quedando únicamente la de Gijón. En la ciudad se mantuvieron algunas instalaciones auxiliares, como las baterías de cok, además de la nueva mini-acería creada en la primera fase de la reconversión. La evolución del empleo durante este proceso de restructuración de Ensidesa da cuenta de la magnitud del ajuste exigido que, a su vez, conllevó el cierre de multitud de pequeñas empresas dependientes de las grandes, provocando una fuerte pérdida de empleo a mediados de la década de los noventa (figura 1$)^{2}$.

Por su parte, otras grandes empresas industriales de la comarca se encontraron también inmersas en un proceso de reestructuración, siendo la década de los noventa un momento de fuerte penetración del capital multinacional; Asturiana de Zinc pasó a manos de Xstrata, Endasa se integró en Inespal (más tarde Alcoa), Enfersa en Fertiberia, Cristalería Española en Saint-Gobain, sin olvidar el desembarco en el área de la química Du Pont. En todo caso, la buena marcha de muchas de estas grandes empresas y de algunos indicadores socio-demográficos de Avilés y su comarca a finales de la década anticipaba el escenario de recuperación que se describe en la sección siguiente y que puede observarse en la figura 1.

Figura 1

EVOLUCIÓN DEL EMPLEO ENAVILÉS, 1990-2010

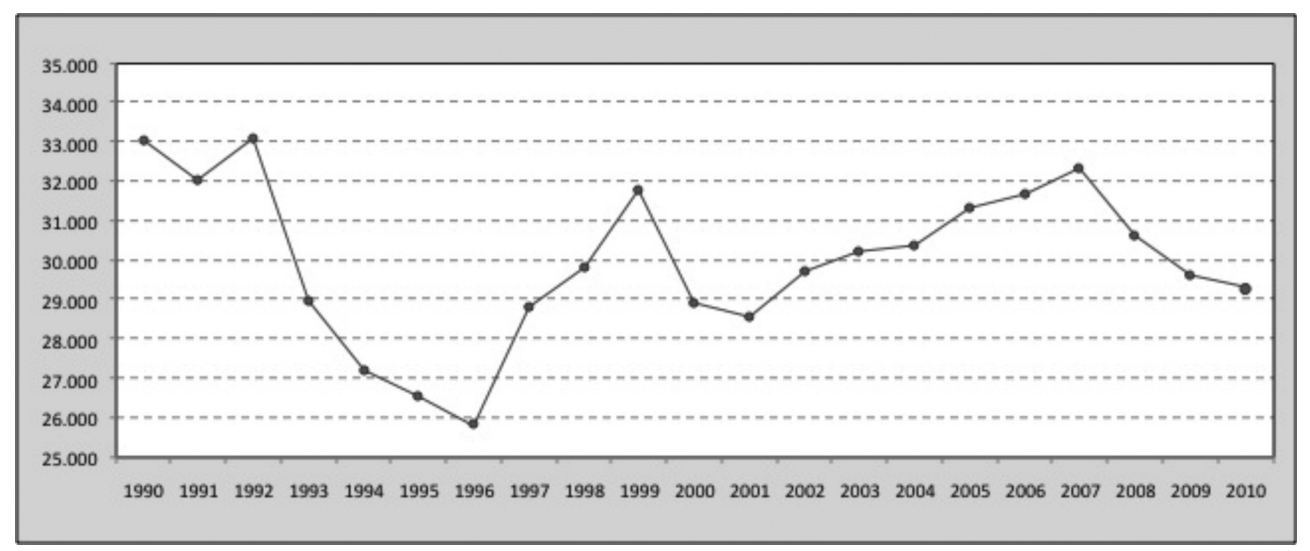

Fuente: Sociedad Asturiana de Estadística.

2 Los datos de la figura 1 aluden a la evolución del empleo y no al número de afiliados. 
Aunque el proceso de desindustrialización descrito estaba sin duda en el origen del declive urbano de Avilés, este fue progresivamente desbordando el ámbito industrial y componiendo un cuadro complejo de efectos que se retroalimentaban en los planos económico, social y territorial-ambiental:

- A la pérdida de competitividad, desinversión, ajustes y cierres de empresas sobrevino la destrucción del empleo y el aumento del paro. Durante los años noventa se perdieron en Avilés 6.714 empleos en la industria, empleando ésta en el año 2000 un 53\% menos que en 1990 (Ayuntamiento de Avilés, 2006). En 1997 el número de parados en el conjunto de sectores alcanzó los 12.000 trabajadores (tasa de paro en torno al 18\%), un 16\% de los desempleados de toda Asturias (De la Madrid, 1999).

- Se evidenció igualmente la tendencia a la despoblación y el progresivo envejecimiento de la población. Tras 15 años de fuerte crecimiento (1950-1965) comenzó a notarse una ralentización en la natalidad y un descenso de los flujos migratorios (Morales, 1982), que a partir de la década siguiente se hizo más profunda y supuso las primeras pérdidas de población. Esto ocurrió especialmente en los barrios obreros, destacando el caso de Llaranes, que tan sólo entre los años 1990 y 1994 perdió el 10,7\% de su población, a pesar de las políticas de prejubilaciones y jubilaciones que consiguieron mantener a parte de sus residentes. El desánimo y el descontento se generalizaron entre la población local, desactivando en gran medida el fuerte tejido asociativo heredado de la época de Ensidesa y alimentando problemas sociales como el alcoholismo, el consumo de drogas y la prostitución (De La Madrid, 1999).

- En el plano espacial también se hizo patente la desarticulación del complejo industrial-portuario de la ría de Avilés, ante la obsolescencia de la infraestructuras productivas y la proliferación de vacíos y ruinas industriales, lo que agravó la situación de la ciudad, que sufrió en aquellos años una merma significativa de sus capacidades funcionales (Benito y López, 2008). A ello hay que sumar la aparición entonces de áreas marginales de chabolismo en la periferia urbana.

- Por último, en el plano ambiental, como consecuencia del desordenado crecimiento industrial y urbano que vivió la ciudad, se produjo un fuerte deterioro. La proximidad de las fábricas a núcleos densamente poblados y la falta de control sobre sus actividades ocasionaron durante décadas importantes problemas de contaminación en la atmósfera, la ría y los suelos de Avilés, haciendo de su calidad medioambiental una de las peores de España y provocando frecuentes problemas de salud a la población (Morales, 1982: 271-273).

\section{AVILÉS: DEL DECLIVE A LA RECUPERACIÓN SELECTIVA}

Tras dos décadas de ligeras pérdidas de población, que supusieron un $4 \%$ y alrededor de 3.400 habitantes entre 1981-2001, en los últimos años la ciudad ha vuelto a registrar cierta recuperación, reflejo de un balance migratorio que compensa con creces un crecimiento natural de sentido negativo, lo que se tradujo en un incremento del 1,3\% entre 2001-2009, que también se trasladó a los municipios integrantes de su comarca funcio- 
$\mathrm{nal}^{3}$ (tabla 1). Aunque se trata de un dinamismo inferior al registrado por las ciudades de Oviedo $(11,4 \%)$ y Gijón $(4,2 \%)$, más diversificadas desde el punto de vista funcional, supone un punto de inflexión en su trayectoria reciente y un indicador de otras transformaciones más sustanciales que han tenido lugar en la última década.

En primer lugar, tras el fuerte retroceso en el volumen de actividad y empleo a consecuencia de la reconversión del cluster siderometalúrgico, las cifras de empresas y empleos también han registrado una evolución netamente positiva desde comienzos de la década y hasta el inicio de la crisis económica en 2007 (tabla 2). Pese a resultar de nuevo inferior al dinamismo de las dos mayores ciudades de la región, entre 2000 y 2006 Avilés aumentó un 9,2\% su volumen de empleo, si bien las pérdidas ocurridas en los tres años posteriores $(-15,4 \%)$ han superado esas ganancias y han supuesto una peor evolución respecto a Gijón $(-7,6 \%)$ y Oviedo $(+5,8 \%)$.

Tabla 1

EVOLUCIÓN DE LA POBLACIÓN EN AVILÉS Y LAS PRINCIPALES CIUDADES DE ASTURIAS

\begin{tabular}{|l|r|r|r|r|r|r|}
\hline \multicolumn{1}{|c|}{ Ciudades } & \multicolumn{1}{|c|}{$\mathbf{1 9 8 1}$} & $\mathbf{1 9 9 1}$ & $\mathbf{2 0 0 1}$ & $\mathbf{2 0 0 9}$ & $\begin{array}{c}\text { Evolución } \\
\mathbf{1 9 8 1 - 2 0 0 1} \\
\mathbf{( \% )}\end{array}$ & $\begin{array}{c}\text { Evolución } \\
\mathbf{2 0 0 1 - 2 0 0 9} \\
(\mathbf{\%})\end{array}$ \\
\hline Avilés & 86.584 & 84.582 & 83.185 & 84.242 & $-3,93$ & 1,27 \\
\hline Comarca Avilés* & 126.753 & 124.159 & 122.679 & 124.100 & $-3,21$ & 1,16 \\
\hline Gijón & 255.969 & 260.267 & 266.419 & 277.554 & 4,08 & 4,18 \\
\hline Oviedo & 190.123 & 204.276 & 201.154 & 224.005 & 5,80 & 11,36 \\
\hline Asturias & 1.127 .007 & 1.098 .725 & 1.062 .998 & 1.085 .289 & $-5,68$ & 2,10 \\
\hline * Concejos de Avilés, Castrillón, Corvera de Asturias e Illas \\
\hline
\end{tabular}

Fuente: INE. Censos y Padrones de población.

Tabla 2

EVOLUCIÓN DE EMPRESAS Y EMPLEOS EN AVILÉS Y LAS PRINCIPALES CIUDADES DE ASTURIAS

\begin{tabular}{|l|r|r|r|r|r|r|}
\hline \multirow{2}{*}{\multicolumn{1}{c|}{ Ciudades }} & \multicolumn{3}{|c|}{ Total empresas } & \multicolumn{3}{c|}{ Total afiliados } \\
\cline { 2 - 7 } & $\mathbf{2 0 0 0}$ & $\mathbf{2 0 0 6}$ & $\mathbf{2 0 0 9}$ & \multicolumn{1}{c|}{$\mathbf{2 0 0 0}$} & \multicolumn{1}{c|}{$\mathbf{2 0 0 6}$} & \multicolumn{1}{c|}{$\mathbf{2 0 0 9}$} \\
\hline Avilés & 2.539 & 2.796 & 2.586 & 24.872 & 27.163 & 22.987 \\
\hline Comarca Avilés* & 3.342 & 3.751 & 3.508 & 39.050 & 44.979 & 40.583 \\
\hline Gijón & 8.671 & 10.018 & 9.622 & 69.986 & 84.842 & 78.374 \\
\hline Oviedo & 8.853 & 10.148 & 9.923 & 104.908 & 122.051 & 129.130 \\
\hline Asturias & 33.594 & 39.069 & 37.747 & 339.057 & 392.310 & 380.896 \\
\hline * Concejos de Avilés, Castrillón, Corvera de Asturias e Illas \\
\hline
\end{tabular}

Fuente: Tesorería General de la Seguridad Social.

3 No existe una única delimitación comarcal, si bien todas coinciden en el caso de los municipios de Avilés, Castrillón, Corvera de Asturias e Illas. Mayores dudas ofrece en cambio la inclusión de Gozón, que en nuestra opinión presentaría un comportamiento demográfico diferenciado, vinculado al dinamismo del núcleo turístico de Luanco. 
Reflejo complementario de esa misma tendencia puede ser la cifra de desempleados registrados en las oficinas del Instituto Nacional de Empleo (INEM), que en Avilés eran 4.787 al finalizar el año 2001 para reducirse a 4.539 en 2008 (el nivel más bajo de la década) y aumentar hasta 6.987 en 2010 . No obstante, ese incremento en apenas dos años $(49,3 \%)$ resultó en este caso inferior al padecido por Gijón $(58,1 \%)$ y, sobre todo, Oviedo $(62,0 \%)$, donde la mayor importancia de la construcción y algunos servicios al consumo agravaron sus efectos. Se ponen así de manifiesto algunas debilidades que aún mantiene la base productiva de Avilés, en relación con el peso de las estructuras heredadas y su influencia sobre una trayectoria local que se transforma de forma lenta.

En el lado positivo, la antigua ciudad industrial fordista ha diversificado su economía, pero sin renunciar a un pasado manufacturero ahora renovado, que aún genera casi la cuarta parte del empleo local (frente al 16\% en Asturias, el 15\% en Gijón y apenas el 7\% en Oviedo), reforzando incluso su posición en la última década por su mejor comportamiento frente a la actual crisis (tabla 3). La primacía del sector terciario (63\% de los ocupados) sigue basada en el predominio del comercio y los servicios al consumo (10.402 empleos). Pero el dinamismo del puerto y la creciente demanda de servicios por parte de las empresas aumentan la presencia de lo que Halbert (2005) denomina actividades de intermediación, que gestionan la circulación tanto de los flujos materiales (mercancías, personas) e inmateriales (capital, información, conocimiento) que articulan el sistema (3.841 empleos), pese a la competencia de los dos grandes centros urbanos próximos.

Tabla 3

ESTRUCTURA DEL EMPLEO POR SECTORES EN LA CIUDAD AVILÉS

\begin{tabular}{|l|r|r|r|r|}
\hline \multirow{2}{*}{ Sectores } & \multicolumn{1}{c|}{ Número de afiliados } & \multicolumn{2}{c|}{ \% total } \\
\cline { 2 - 5 } & \multicolumn{1}{|c|}{$\mathbf{2 0 0 0}$} & \multicolumn{1}{c|}{$\mathbf{2 0 0 9}$} & \multicolumn{1}{c|}{$\mathbf{2 0 0 0}$} & \multicolumn{1}{c|}{$\mathbf{2 0 0 9}$} \\
\hline Agrario & 401 & 235 & 1,61 & 1,02 \\
\hline Industrial & 4.539 & 5.582 & 18,25 & 24,28 \\
\hline - Siderometalurgia & 1.449 & 1.742 & 5,82 & 7,57 \\
\hline - Resto del sector industrial & 3.090 & 3.816 & 12,43 & 16,61 \\
\hline Construcción & 4.335 & 2.672 & 17,43 & 11,62 \\
\hline Servicios & 15.597 & 14.522 & 62,71 & 63,17 \\
\hline - Servicios al consumo y personales & 11.085 & 11.844 & 44,56 & 51,52 \\
\hline - Servicios basados en conocimiento* & 4.200 & 2.399 & 16,88 & 10,43 \\
\hline - Actividades culturales & 312 & 255 & 1,25 & 1,11 \\
\hline Total & 24.872 & 22.987 & 100,00 & 100,00 \\
\hline * Según tablas de conversión CNAE-93 y CNAE-2009, el dato del año 2000 incluye las Actividades Veterinarias \\
y las actividades de Empresas de Trabajo Temporal, no incluidas en 2009. & \\
\hline
\end{tabular}

Fuente: Tesorería General de la Seguridad Social. 
Pese a todo, Avilés sigue enfrentándose a ciertas desventajas comparativas heredadas que aún limitan su capacidad para convertirse en una ciudad con una economía basada en el conocimiento (VanWinden et al., 2007; Musterd et al., 2007) desde varios puntos de vista. Si se consideran los sectores que suelen integrarse hoy bajo esa denominación (industrias de alta intensidad tecnológica, servicios a las empresas intensivos en conocimiento, otros servicios como educación y salud, junto a las industrias culturales), en Avilés apenas emplean a uno de cada ocho trabajadores (12,3\% de los afiliados), cifra bastante inferior a las de Gijón $(18,4 \%)$ y Oviedo $(36,1 \%)$, con una presencia muy superior de servicios avanzados a las empresas (2.399 afiliados en 2009, el 10,43\% del total). Resulta también escasa la presencia del sector cultural, 255 afiliados, es decir tan sólo el 1,11\% de los ocupados, pese a haberse convertido en una de las apuestas de futuro, influida por la atención que ahora se presta a la construcción de ciudades creativas (Scott, 2006). Incluso desde un punto de vista dinámico se advierte cómo los mayores incrementos entre 2000 y 2009 se localizaron en la siderometalurgía de municipios de la comarca (Castrillón y Corvera), mientras que las mayores caídas afectaron a los servicios avanzados a las empresas, generalizadas pero más importantes en el caso de la ciudad de Avilés.

Lo mismo ocurre con su stock de capital humano según el último censo, pues mientras los ocupados con titulación universitaria eran el 21,0\% en Avilés, suponían el 24\% en Gijón y hasta el 33,5\% en la capital regional. Idénticas diferencias se reproducen al considerar la presencia de los grupos profesionales calificados por Florida (2002) como clase creativa, que aquí suponen un 30,8\% del total, por un 34,6\% en Gijón y el 43,6\% en Oviedo.

No obstante, la existencia de una base industrial que, al menos en parte, ha llevado a cabo un intenso proceso de innovación también se traduce en resultados positivos. Por ejemplo, las empresas de Avilés consiguieron acceder a créditos para proyectos de innovación financiados por el Centro para el Desarrollo Tecnológico Industrial (CDTI) del Ministerio de Industria por un total de 15,9 millones en el periodo 2000-2006, lo que equivale a un promedio de 569,7 euros por cada empresa localizada en la ciudad en esa última fecha, muy por encima de Gijón $(300,8)$ y, sobre todo, de una ciudad de servicios como Oviedo $(46,9)$. Al mismo tiempo, en Avilés se generaron 10,7 patentes por cada mil empresas en ese mismo periodo, frente a 10,3 en Gijón, mientras las 15,9 de Oviedo guardan relación directa con su carácter de capital y principal sede universitaria regional (Méndez y Sánchez Moral, 2010). Por otro lado, se confirma el buen comportamiento del indicador a escala comarcal, impulsado por la presencia de la empresa Du Pont en el municipio de Corvera.

Un último factor del proceso de revitalización lo ofrece la evolución del precio medio de la vivienda durante esta última década. Aunque en 2010 Avilés continúa siendo una ciudad con suelo más barato, 653 euros por metro cuadrado frente a 694 en Oviedo y 740 en Gijón, el incremento registrado desde el año 2000 (50,5\%) superó con creces el de las otras dos (7,9\% y 39,6\% respectivamente). La resistencia a la caída de precios desde 2007 también ha sido mayor $(-11,8 \%)$ que en los casos ovetense $(-14,1 \%)$ y gijonés $(-20,3 \%)$, lo que parece confirmar la mejora de su valoración como espacio para residir. 


\section{CLAVES DE LA REGENERACIÓN URBANA: LA CONSTRUCCIÓN DE UN NUEVO MILIEU INSTITUCIONAL}

Desde hace ya más de una década, Avilés experimenta un proceso de revitalización económica y regeneración urbana que le ha permitido abandonar la espiral negativa que alimentaba el declive urbano. Aunque en un proceso de este tipo influyen factores múltiples, aquí se centrará la atención en el protagonismo de unos actores con características e intereses heterogéneos, pero que se han implicado de diferentes modos en el desarrollo local, han movilizado recursos existentes en la ciudad o los han generado y, de forma progresiva, han establecido tanto redes internas de colaboración para fines concretos que no eliminan el conflicto, pero buscan formas de resolución más dialogadas, como redes de carácter global, que permiten conectar hoy la ciudad con el exterior.

Todo ello se ha reflejado en un aumento de las acciones innovadoras, tanto en los planos económico como social, que incluyen «la voluntad de invertir en la creación o el desarrollo de empresas, implicarse en iniciativas locales o regionales de gobernanza, e inventar nuevas formas de intervención para contribuir al desarrollo socioeconómico de la colectividad» (Fontan, Klein y Tremblay, 2005: 17). La multiplicación de actores implicados (figura 2) y la aparición de coaliciones para el desarrollo de la ciudad son el primer aspecto a destacar.

Los actores externos continúan desempeñando una importante función en la evolución de la ciudad, tanto en relación con las diferentes políticas (sectoriales, regionales y horizontales) que la afectan, como a partir de las decisiones estratégicas que toman las empresas públicas dependientes del gobierno central y las grandes corporaciones transnacionales que cuentan con algún establecimiento en Avilés.

Por una parte, la ciudad se ha visto beneficiada por un significativo volumen de fondos europeos, cifrados en cerca de 400 millones de euros, procedentes del FEDER y su participación en diversas acciones o programas (Urban, Equal, Interreg, IFOP ...), destinados a actuaciones tan importantes como la mejora del puerto y otras infraestructuras básicas, la rehabilitación urbanística o la gestión de residuos, entre otras. Más recientemente se habría obtenido financiación a través del FSE para programas de atención a familias en riesgo de exclusión social.

En el caso del gobierno central, las diversas políticas industriales aplicadas desde el plan de reconversión de 1981 han tenido una incidencia directa sobre sectores estratégicos para la ciudad, al tiempo que empresas públicas como la Autoridad Portuaria, SEPI-Infoinvest o RENFE han protagonizado la ampliación del puerto, la promoción del nuevo Parque Industrial Principado de Asturias (PEPA) sobre la antigua cabecera siderúrgica, o los actuales proyectos de cambio en los accesos ferroviarios, de especial importancia para la transformación urbana emprendida. El gobierno regional asturiano forma parte de las redes tejidas para financiar algunos de los proyectos emblemáticos de los últimos años (Centro Cultural Niemeyer, Isla de la Innovación...), pero como veremos también ha puesto en práctica otras acciones de las que se ha beneficiado la ciudad como la red de centros de servicios avanzados tecnológicos (SAT) o la creación de consorcios como el destinado a la gestión de los residuos sólidos (Cogersa). Finalmente, buena parte de la historia industrial reciente de Avilés ha estado ligada a esas cinco grandes empresas transnacionales mencionadas anteriormente, cuya decisión de mantener y modernizar sus fábricas, sin llevar a cabo por el momento nue- 
vas deslocalizaciones, sigue siendo esencial para el futuro de la ciudad, tanto por el empleo directo generado como, sobre todo, por sus efectos de arrastre sobre la industria auxiliar; sin olvidar una capacidad para fijar población en Avilés que justificaría la promoción de determinados equipamientos y servicios urbanos.

Figura 2

ACTORES PÚBLICOS Y PRIVADOS IMPLICADOS EN LA REGENERACIÓN DE AVILÉS

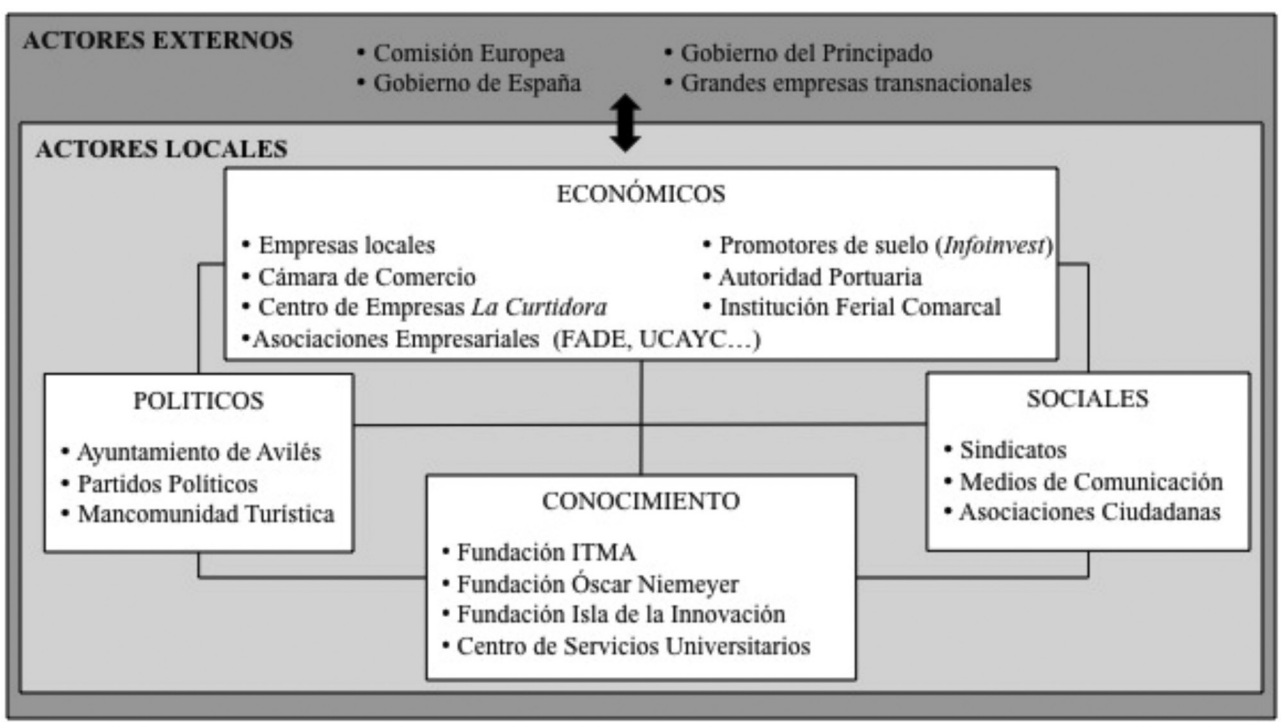

Fuente: Elaboración propia.

No obstante, el cambio más relevante de estos años ha sido la consolidación de un conjunto de actores políticos, económicos, sociales y ligados al conocimiento o la cultura cuyas decisiones responden, sobre todo, a claves locales. Aunque algunos de ellos tienen larga tradición (gobierno local, cámara de comercio, sindicatos...), sólo desde la pasada década han ampliado sus funciones para incorporar proyectos de desarrollo y en esa tarea convergen hoy con otros de origen más reciente, surgidos a menudo como respuesta frente al declive (asociaciones empresariales, centro de empresas, mancomunidad turística, centro tecnológico, fundaciones...).

Entre todos ellos, el Ayuntamiento de Avilés ha adquirido un notorio protagonismo, tanto por la generación de numerosas iniciativas que pueden calificarse de innovadoras, como por su función de agente catalizador del desarrollo. Pero junto con ello, se evoluciona hacia una gobernanza más participativa basada en una movilización y una inversión en capital social que se traduce en la construcción de múltiples redes locales, informales o ya formalizadas, que complementan las que la ciudad mantiene con el exterior.

En ese sentido, aunque la ciudad de Avilés no cuenta formalmente con un organismo de concertación entre el gobierno local y los agentes sociales, esa finalidad inspira los sucesivos pactos locales suscritos en los últimos años y enmarcados en iniciativas similares a escala regional: fundamentalmente el pacto Avilés por el Empleo 2000-2003 (estrategia ampliada al 
periodo 2004-2008), firmado en el contexto del Pacto Institucional por el Empleo en Asturias (2000) o el ya mencionado Pacto Local Avilés Avanza, enmarcado en el Acuerdo para la Competitividad, el Empleo y el Bienestar en Asturias (periodo 2008-2011).

\section{ACCIONES RECIENTES DE REVITALIZACIÓN EN LA CIUDAD DE AVILÉS}

Liderados por el Ayuntamiento, los actores locales han puesto en marcha una serie de estrategias que pueden agruparse en función de si tienen como objetivo: la promoción económica y del empleo, buscando crear una ciudad competitiva; la promoción de la innovación y la cultura, o fomento de una ciudad inteligente; la mejora de la calidad de vida y del medioambiente, en aras de una ciudad más habitable; o el reforzamiento del sistema local, teniendo como meta poner en marcha una ciudad con proyecto.

\section{La ciudad competitiva}

En el primer caso, destacan las operaciones encaminadas a aumentar la competitividad de la ciudad, maltrecha tras el período de declive y en peor situación que Oviedo y Gijón. Junto con la mejora general de accesos y comunicaciones en el interior o con el exterior, destaca concretamente la ampliación del puerto, uno de los proyectos urbanos recientes más importantes y cuyo ordenamiento corresponde a la Autoridad Portuaria, a la que el PGOU encomienda la labor de organizar este espacio «de modo funcional y en sinergia con el desarrollo urbano del entorno» (Ayuntamiento de Avilés, 2005). Para aprovechar el potencial del puerto (se estima que éste representaría en torno a un 5\% del PIB regional) los planes de futuro pasan por ampliar la línea de atraque en la margen derecha, ganando con ello superficie para nuevas zonas de servicios, concesiones de suelo público, viales, etc. Esto permitiría hacer bascular la actividad portuaria hacia esa área, permitiendo reforzar en el sector más próximo al centro de la ciudad nuevas potencialidades en un puerto dedicado principalmente a la carga de materiales industriales. En esta estrategia se inscribe la construcción de una nueva lonja, la adecuación de un área deportiva o la desafección de terrenos para la apertura del paseo marítimo o en el sector del Centro Niemeyer.

En segundo lugar, cabe mencionar una serie de acciones que, desde la década de los noventa, tratan de paliar los efectos del declive industrial sufrido, a partir de inversiones en suelo industrial (Polígono «Las Arobias»), del fomento de los servicios a empresas y la natalidad empresarial (Centro de Empresas «La Curtidora»), o de los servicios para las industrias del sector siderúrgico y la implantación de una cultura basada en la I+D+i («Centro Tecnológico del Acero»). De todas ellas, resulta ya emblemática la promoción del PEPA en 1992, que cuenta con una superficie de más de 2 millones de metros cuadrados liberados de la cabecera siderúrgica de Ensidesa, en una obra de enorme complejidad que arrancó con la descontaminación de los suelos y que posteriormente tuvo que simultanear trabajos de derribo y mantenimiento de algunas instalaciones en uso, antes de proceder a su reurbanización por medio de Gestión de Suelo de Ensidesa Sociedad Anónima (Gesesa), creada en 1998 por SEPI a través de su filial Infoinvest.

El parque empresarial, que hasta el momento habría generado más de 3.000 puestos de trabajo y de una inversión de más de 104 millones de euros, con una aportación impor- 
tante de los Fondos FEDER, ha contribuido a la diversificación económica y al aumento del tejido de PYMEs en la ciudad. De hecho, dentro del proyecto se reservó una parte de suelo industrial para empresas de tamaño medio y grande, que se ocupó rápidamente, y otra para pequeñas empresas, cuya venta ha sido más lenta. El perfil de las nuevas actividades instaladas sigue siendo hoy un motivo de controversia, en relación con el papel que debe jugar la industria en el futuro de la ciudad y las estrategias de comercialización del suelo de Infoinvest, así como con el origen de las empresas, varias de ellas relocalizadas desde otras ubicaciones dispersas por el término municipal de la ciudad y que no suponen añadir nuevas actividades. Por otro lado, la Cámara de Comercio de Avilés promovió una serie de naves-nido en el PEPA con la intención de fomentar la natalidad empresarial. Resultado de todo ello nos encontramos una mezcla de empresas, en un elevado porcentaje dedicadas a la fabricación y el suministro a otras empresas de la comarca, donde con frecuencia mantienen otros establecimientos, junto a empresas comerciales y concesionarios, cuya presencia en el PEPA parece menos justificable.

Con todo, a día de hoy estaría desarrollado el $70 \%$ del suelo, correspondiendo el $20 \%$ restante al sector de las baterías de cok y quedando otro $10 \%$ aún por desarrollar. En paralelo se han mejorado los accesos desde la autopista y los viales de servicio con la zona portuaria, en un sector que es especialmente interesante por los planes de creación allí de una zona logística con las obras de ampliación del Puerto.

Finalmente, el Programa Territorial de Formación y Empleo toma como prioridad la creación de oportunidades de empleo para los grupos más vulnerables, estableciendo cinco ejes de trabajo en torno a formación y cualificación del empleo, orientación e intermediación laboral, fomento de la calidad del empleo, de la igualdad de oportunidades y del desarrollo local.

\section{La ciudad inteligente}

Pese al fuerte dinamismo empresarial reflejado en el anterior análisis de los datos, es en la cuestión de la innovación y la capacidad emprendedora donde los entrevistados detectaron algunos de los principales déficits. En este sentido, varios entrevistados señalaron la mala conexión entre los centros tecnológicos, las empresas y la universidad regional, y la escasa capacidad emprendedora de los ciudadanos avilesinos, en parte como consecuencia de las décadas bajo el «paraguas» de las empresas estatales, así como de las condiciones laborales que se derivaron de su desmantelamiento, pues se tradujeron en elevadas prejubilaciones que en unos casos impidieron y en otros desincentivaron la capacidad emprendedora de esos trabajadores industriales, cuya experiencia y conocimiento no supo ser aprovechada por la ciudad. En todo caso, resulta innegable el intento reciente por parte del gobierno local por corregir la situación (Ayuntamiento de Aviles, 2008).

En primer lugar, se trabaja en el fomento de la «cultura emprendedora», cuestión que, en colaboración con el Gobierno del Principado, se introduce ya desde el plan de estudios de la enseñanza primaria y secundaria obligatoria. A ello hay que añadir la puesta en marcha de instrumentos para la creación de nuevas empresas (primer punto de asesoramiento y tramitación de toda Asturias, semillero de empresas en «La Curtidora», microcréditos de ASTUR$G A R \ldots$... El apoyo se mantendría a lo largo del ciclo de vida de la empresa, destacando 
especialmente la línea de apoyo técnico a la internacionalización de las PYMEs de Avilés en colaboración con Sociedad de Promoción Exterior Principado de Asturias (ASTUREX), la Federación Asturiana de Empresarios (FADE) y la Cámara de Comercio de Avilés.

Por lo que respecta a la cultura, del conjunto de medidas emprendidas destaca, muy por encima del resto, el emblemático proyecto del Centro Cultural Internacional Oscar Niemeyer, cedido al Principado de Asturias por el arquitecto Oscar Niemeyer en agradecimiento por la concesión del Premio Principe de Asturias de las Artes en 1989 e inaugurado recientemente. El Centro está situado en la llamada Isla de la Innovación, cuyo desarrollo urbanístico se comenta en la sección siguiente. En todo caso, este no sería el único ejemplo de promoción de infraestructuras específicamente culturales, pudiendo mencionar la creación de centros de arte, un museo de la ciudad, una escuela de música, otra de cerámica o un conservatorio.

La actual estrategia de city-marketing aprovecha el empuje que ha supuesto ese primer proyecto para situar a Avilés en el «mapa» de ciudades con proyección internacional, atrayendo personalidades famosas a la ciudad para ayudar a la promoción de otros aspectos presentes históricamente en Avilés, como la cultura, la música, las artes escénicas, el turismo o la gastronomía ${ }^{4}$. Multitud de iniciativas dan cuenta del esfuerzo realizado en la última década, permitiendo la consolidación de un producto turístico en torno a la cultura ${ }^{5}$, como el Plan de Turismo Gastronómico, la recuperación del Teatro Palacio Valdés o el Certamen Nacional de Cortos. De forma complementaria, la ciudad habría adecuado en estos años su infraestructura turística, pasando el número de hoteles y restaurantes respectivamente de 6 a 10 y de 47 a 75, tan sólo entre 2006 y 2009.

\section{La ciudad habitable}

Del conjunto de acciones encaminadas hacia la mejora de la calidad de vida y la sostenibilidad urbana cabe recordar, antes que nada, el decisivo trabajo de saneamiento de la ría de Avilés, con una obra que comienza en 2003 y que ha supuesto una inversión de más de 14 millones de euros y la retirada de 170.000 metros cúbicos de lodos contaminados. Otras acciones como la integración en consorcios regionales para la gestión de residuos urbanos, suministro de aguas o transporte de manera unitaria, apostando por un servicio único y menores costes a través de fórmulas de cooperación con otros municipios y ciudades, son igualmente consideradas de interés de cara a la elaboración de la futura Agenda 21 de Avilés (Ayuntamiento de Aviles, 2008).

4 El denominado «efecto Niemeyer» ha supuesto, según informaban algunas noticias durante los últimos meses, la visita al Centro Niemeyer de más de 400.000 personas en los primeros tres meses de funcionamiento del mismo. Para la propia ciudad ha supuesto duplicar en 2011 el número de consultas en la oficina de turismo respecto a 2010 durante julio (10.139 frente a 5.661) y las fiestas patronales de agosto (10.139 frente a 5.661), o a cuadriplicarlas en Semana Santa (3.619 frente a 913).

5 Una idea que de forma recurrente aparece en las entrevistas alude a la existencia de un sustrato cultural importante en el pasado de la ciudad, lo que en tiempos le valió a Avilés el apelativo de la «Atenas del Norte». Su origen estaría en las inversiones realizadas por los emigrantes retornados («indianos»), sobre todo a finales del siglo XIX, para mejorar la vida cultural de la ciudad. Para algunos durante los años de la dictadura franquista se trató de borrar este legado cultural, al cual parece que la ciudad apela de nuevo. 
La política de rehabilitación del centro histórico, elemento destacado de las actuaciones del consistorio avilesino desde los años noventa, se ha visto reforzado por iniciativas más recientes, muchas de ellas apoyadas desde la Unión de Comerciantes de Avilés y su Comarca (Ucayc), como la peatonalización, el fortalecimiento de la presencia del pequeño comercio en el centro o la mejora de los sistemas de gestión del acceso y el estacionamiento. De acuerdo con las estimaciones del Ayuntamiento de Avilés realizadas ex profeso para esta investigación, el consistorio habría invertido desde 1995 en torno a 11 millones de euros en la renovación de infraestructuras, reurbanización y peatonalización de las calles del conjunto histórico. A ello hay que sumar las subvenciones para la recuperación de los edificios de propiedad privada, cuya cuantía entre el año 2003 y 2010 asciende a 1,4 millones de euros. En consecuencia, se ha producido un alza importante de precios en la vivienda del centro de Avilés, donde han prosperado también actividades ligadas a la restauración y el turismo en general, siguiendo un proceso similar al ocurrido en otras ciudades.

Junto con ello, se habría realizado un esfuerzo importante de mejora de los equipamientos, a través de una «política de barrios» encargada de subsanar carencias y convertir a Avilés en una de las ciudades con mejor disposición de servicios públicos.

Mención especial merece la coordinación de las acciones en distintas áreas como Salud, Infancia, Igualdad o Juventud, dentro de un Plan Rector de políticas para la cohesión social del municipio, que tiene en las políticas activas de vivienda un pilar esencial y que daría continuidad a la labor internacionalmente reconocida del Programa Municipal de Erradicación del Chabolismo, Realojamiento e Integración Social de la Población, iniciado a finales de los ochenta. De hecho, Avilés fue premiada en la calificación del BEST del VI Concurso de Naciones Unidas sobre Buenas Prácticas para Mejorar las Condiciones de Vida (2006).

\section{La ciudad con proyecto}

Concluimos este breve repaso con el conjunto de políticas que tratan de reforzar el sistema local y constituir una ciudad con proyecto. Un proyecto en el que se intensifican las sinergias entre los elementos arriba comentados por separado y que tiene en la reciente aprobación del principal documento de planeamiento de la ciudad, el Plan General de Ordenación Urbana de Avilés (en adelante PGOU), un excelente ejemplo del esfuerzo realizado en esta dirección y que es motivo de análisis en el apartado final.

En síntesis, todo apunta a que, en efecto, la cooperación es ahora el substrato en que han germinado la mayor parte de las iniciativas ya comentadas y que han transformado la ciudad en estos últimos años, tal como ocurre con algunas orientadas a su dinamización económica (PEPA, Mancomunidad Turística Comarca de Avilés, Pacto Local Avilés Avanza, Centro de Empresas La Curtidora, Institución Ferial Comarcal-Ifecav) o a lograr una mejor inserción en la sociedad del conocimiento (Fundación ITMA y Centro Tecnológico del Acero, Fundación Oscar Niemeyer, Fundación Isla de la Innovación). Resulta significativo que el Ayuntamiento está presente entre los socios que participan en esas ocho iniciativas, el gobierno regional lo está en cinco y el gobierno central en cuatro, mientras la Cámara de Comercio y la Autoridad Portuaria lo están en tres, siendo menor la presencia de los restantes actores. Eso también demuestra la importante colaboración del Estado en las estrategias de promoción y la lenta incorporación de los actores privados, casi siempre limitada a los proyectos 
destinados a la dinamización de la actividad económica. Al tiempo, Avilés también se ha integrado en numerosas redes internacionales de ciudades (a modo de ejemplo: International Association of Education Cities, Conference of Atlantic Arc Cities, Cool Cities, Spain Convention Bureau...) destinadas al intercambio de experiencias en diferentes ámbitos relacionados con el desarrollo económico o social principalmente.

La construcción de toda esta arquitectura institucional está en la base de las principales acciones llevadas a cabo para impulsar el resurgimiento de la ciudad. No obstante, el trabajo de campo realizado ha puesto igualmente de manifiesto una reivindicación clave para muchos de los actores entrevistados, como es la necesidad de cambiar el enfoque «local» a favor de una óptica comarcal, sobre todo en aspectos como la gestión de servicios comunes o la planificación de las infraestructuras y del suelo industrial. En este sentido, si bien existen algunas experiencias previas destacables, como la mencionada Mancomunidad Turística o la Ucayc, inciativas como el Observatorio Socioeconómico Comarcal o la Ifecav, tendrían en cambio un papel, en principio, más difícil de identificar. Más aún, en algunos otros casos hablamos de propuestas de coordinación territorial en la escala comarcal, sin duda muy interesantes pero aún por concretar, como el Plan Rector Territorial para la Comarca de Avilés, con la participación del Ayuntamiento la ciudad, el Principado y los concejos de la comarca, que se marca como objetivos el programar y ampliar la bolsa de suelo industrial en respuesta a la creciente demanda.

\section{CONSENSOS Y DEBATES EN TORNO AL MODELO DE CIUDAD: EL NUEVO PLAN GENERAL (2007)}

El nuevo planeamiento refleja un proyecto de ciudad que evidencia cómo la tesis de la reindustrialización como única vía para alcanzar el desarrollo urbano, defendida hasta bien entrados los años noventa por los poderes públicos desde la escala local a la nacional, parece definitivamente superada. No obstante, esa visión impregnaba el Plan Estratégico Avilés 2000, elaborado entre 1992 y 1993 por el Ayuntamiento de Avilés y que supone un primer documento de referencia. Dicho Plan se articulaba en torno a la modernización y diversificación de la base industrial de la ciudad, además de la mejora de la oferta comercial, cultural y turística, de la calidad de vida y la participación ciudadana.

De esta forma, la recualificación de 2,5 millones de $\mathrm{m}^{2}$ pertenecientes a la antigua Ensidesa, manteniéndose en ellos el uso industrial en gran medida, daba continuidad a proyectos urbanos como el polígono industrial de «Las Arobias» (1986), que se constituyó como resultado de la declaración de Avilés como Zona de Urgente Industrialización por parte del Gobierno español, o el Centro de Empresas «La Curtidora» (1993), rehabilitado con fondos del Principado y del programa europeo de reconversión de zonas siderúrgicas: RESIDER.

En todo caso, resulta sintomático que el Plan Estratégico nunca se ejecutara y que la sociedad Avilés 2000, participada por las tres administraciones públicas y encargada de la gestión de los suelos desafectados, viera retrasarse su constitución hasta 1998 ante los constantes disensos por razones políticas (De la Madrid, 1999). La falta de visión compartida sobre el futuro de Avilés en un momento que puede ser interpretado como de búsqueda de un modelo de desarrollo urbano propio y alternativo al siderúrgico-industrial que caracterizó el pasado reciente del núcleo y su comarca, afectó al planeamiento urbano, que permanece 
vigente desde el año 1986. Las dificultades para su actualización se agravaron ante la etapa de alternancia que se abrió por aquel entonces en el gobierno local ${ }^{6}$.

El nuevo PGOU, aprobado definitivamente en agosto de 2007, atestigua el cambio de enfoque y el nuevo discurso de los poderes locales sobre la ciudad (Benito, 2004)7 . Tal como señalan algunos de los máximos responsables de la elaboración del PGOU, la ciudad ha sufrido «una reconversión económica, sin reconversión urbana» (Leira y Calvo, 2003); la reindustrialización, tan defendida en la etapa anterior, aunque necesaria, deja de ser por tanto suficiente para la consecución del objetivo de hacer de Avilés una ciudad atractiva, moderna y competitiva. Las nuevas bases del desarrollo socioeconómico incorporan, o al menos refuerzan dentro del «modelo Avilés», dimensiones como el comercio, el turismo, la innovación o la cultura (Benito y López, 2008). De hecho, el nuevo planeamiento apuesta por configurar una doble centralidad apoyada fundamentalmente en las actividades de servicios; centralidad comarcal en la conurbación de casi 140.000 habitantes y centralidad metropolitana compartida con Oviedo y Gijón, en lo que seria una moderna metrópoli en red de carácter polinuclear (Rodríguez, Menéndez Blanco, 2009).

Pero, para alcanzar tales objetivos, resulta necesario superar lo que el Plan General considera una serie de limitaciones arrastrados por una ciudad que, de partida, cuenta con un menor tamaño urbano en el contexto metropolitano, frente a Oviedo y Gijón. Entre esos problemas se alude a la obsolescencia económica de las antiguas instalaciones siderúrgicas, la mayoría de ellas ya demolidas, a la que se suma hoy una obsolescencia territorial que se traduce en la ausencia de un soporte territorial adecuado para la nueva economía y la escasez de proyectos urbanos novedosos (frente a emplazamientos alternativos elegidos con frecuencia dentro del área urbana central de Asturias). A ello contribuiría la degradación medioambiental observada, ante la aparición de suelos «marcados» por el modo de implantación de la gran industria; la falta de planificación en las infraestructuras desarrolladas en su proceso de expansión; la contaminación de suelos, agua (parcialmente corregida), atmósfera y paisajes; o la baja calidad de la vivienda e insuficiencia del sistema de saneamiento. Las tensiones de crecimiento, sobre todo en la comarca, las carencias de ordenación o las condiciones del marco competencial necesitado de una visión más integral en lo sectorial y lo territorial, son algunas otras limitaciones identificadas.

Frente a ello, se reconoce igualmente la existencia de algunas bazas importantes que pueden ser entendidas en tanto que activos o recursos territoriales específicos de la ciudad, lo mismo que el contexto institucional y las formas de relación entre actores públicos y privados, a las que se ha dedicado buen parte de nuestro análisis. Entre ellas se identifica la ría de Avilés, apostando el nuevo planeamiento por un «salto» sobre la misma que haga posible

6 Desde las primeras elecciones democráticas en 1979 el PSOE ha gobernado la ciudad, salvo en la legislatura 1995-1999 donde la falta de acuerdo de los partidos de izquierda dio la victoria al Partido Popular, que gobernó sin mayoría absoluta.

7 El documento de revisión del Plan (2005), elaborado a partir de más de 700 alegaciones presentadas durante el largo proceso de información pública da cuenta del cambio de visión: «Avilés, en el marco más amplio asturiano, ha sufrido un profundo proceso de reconversión económica. No ha hecho sin embargo una transformación urbana correlativa. Afronta un proceso de cambio económico, hoy en curso, sobre la base de un soporte urbano heredado, que es aún el de una ciudad marcada por la siderurgia. No está preparada, pues, en términos urbanos, para ese cambio que, se quiera o no, es profundo, responde a claves distintas a las de la primera industrialización y presenta otras exigencias» (Ayuntamiento de Avilés, 2005). 
el crecimiento urbano hacia el este, poniendo en valor la lámina de agua y favoreciendo la recualificación de los frentes de agua de la ciudad y los terrenos liberados por la industria. De esta forma, la ría se convierte en el auténtico eje de la nueva centralidad, superando su condición de barrera para la ciudad y de separación entre el espacio residencial y el espacio industrial, favoreciendo la unión de ambos por medio de la instalación de nuevas actividades y equipamientos (paseo, espacios de ocio, puerto deportivo, Centro Niemeyer...). Al respecto cabe precisar que aunque el Proyecto Bilbao Ría 2000 es un primer referente para el proceso de transformación de Avilés, otras muchas ciudades han servido de inspiración a la Fundación Metrópoli, a quién se encargó la elaboración del Masterplan de la Isla de la Innovación, como las llamadas «ciudades del agua» (Ámsterdam, Brujas, Estocolmo, Nantes...).

Por su parte, el Puerto, considerado históricamente un motor económico de la ciudad, puede contribuir activamente al proceso de transformación y revitalización urbana gracias a su plan de ampliación y la posibilidad de instalar nuevas funciones y actividades singulares en el sector más cercano a la ciudad. En tercer lugar, y como principal oportunidad para Avilés, se alude a la importante reserva estratégica de suelos liberados por la reconversión de la siderurgia, algo que diferencia sustancialmente el potencial de Avilés frente a ciudades asturianas competidoras como Oviedo o Gijón; su singularidad y posición central en la ciudad y la comarca permiten pensar en complementar parte del suelo destinado a las actividades industriales con la implantación de funciones terciarias avanzadas, equipamientos y usos residenciales. El atractivo del casco histórico, como recurso con un gran potencial para el turismo y el comercio de la ciudad, o la proximidad al aeropuerto por su capacidad para mejorar el posicionamiento a escala nacional, serían igualmente otros aspectos valorados de forma muy positiva.

De forma sintética, los nuevos proyectos urbanos que mayor impacto tendrán sobre la ciudad corresponden al Área de Planeamiento Remitido denominada «Nueva centralidad», cuya complejidad hace que la ordenación de detalle no se incluya en el PGOU y se remita a su desarrollo mediante un Plan Especial ${ }^{8}$. En efecto, se trata de tres ámbitos diferenciados (figura 3); el de la estación de ferrocarril-APR.C-1 (62.200 m²), los terrenos de la antigua Ensidesa, entre el PEPA y la ría, donde se desarrollaría la Isla de la innovación-APR.C-2 (200.370 m²) y el barrio de Divina Pastora-APR.C-3 (309.478 $\mathrm{m}^{2}$ ), que suman una superficie de más de 57 hectáreas y cuyo desarrollo necesitará de la participación de diferentes administraciones; desde el Ministerio de Fomento, la Sociedad Estatal de Participaciones Industriales (SEPI), la Autoridad Portuaria, el Principado de Asturias o el propio Ayuntamiento de Avilés.

Las obras a realizar incluirían la supresión de las barreras existentes que separan la ría de la ciudad, en especial de las ferroviarias ${ }^{9}$, el traslado del aparcamiento de camiones de la

8 En enero de 2010 la Sociedad Isla de la Innovación convocó un concurso internacional para la redacción del Plan Especial, presentándose hasta 18 propuestas y resultando elegida una Unión Temporal de Empresas (UTE) constituida por Taller de Ideas-Ove Arup-Tecnia Ingenieros. En su equipo se encuentran algunos redactores del Masterplan y además, la propuesta viene apoyada por el estudio del arquitecto Norman Foster. Se espera que el documento de Avance del Plan Especial que elaboren pueda ser presentado en febrero de 2011.

9 En diciembre de 2010 el Ministerio de Fomento da a conocer la solución consensuada: una nueva variante ferroviaria, conjunta para servicios de viajeros y mercancías, cuyo trazado discurre prácticamente paralelo a la N-632, así como una nueva Estación Central Intermodal frente a la Isla de la Innovación (a partir de ese punto los planes del Ayuntamiento ya preveían la puesta en servicio de un tranvía urbano que diera servicio hacia el norte de la ciudad). 
zona de Divina Pastora a un nuevo Centro de Transportes próximo al PEPA, la reorganización de los corredores ferroviarios y la construcción de nuevas zonas residenciales en este barrio degradado, que es uno de los puntos más visibles desde los accesos principales a la ciudad. También se pretende mejorar la accesibilidad de la margen derecha, con especial atención a la ordenación de todo el entorno inmediato del Centro Cultural Internacional Oscar Niemeyer, auténtico elemento vertebrador de la ordenación en todo el ámbito, así como el desarrollo de una gran zona terciaria de servicios a empresas y personas en la margen derecha.

Además del PGOU y de este Plan Especial de la Nueva Centralidad de Avilés, se suma al nuevo planeamiento de la ciudad el Plan Especial de Mejora del Casco Histórico de Avilés, aprobado en 2010 y que da continuidad a las distintas iniciativas de protección y rehabilitación emprendidas a lo largo de las últimas décadas. En el momento actual se pretende reforzar las sinergias entre el centro histórico y el proyecto de la Isla de la Innovación, así como sentar las bases para una futura solicitud de declaración del casco histórico como Patrimonio de la Humanidad.

Figura 3

ÁREA DE PLANEAMIENTO REMITIDO: NUEVA CENTRALIDAD

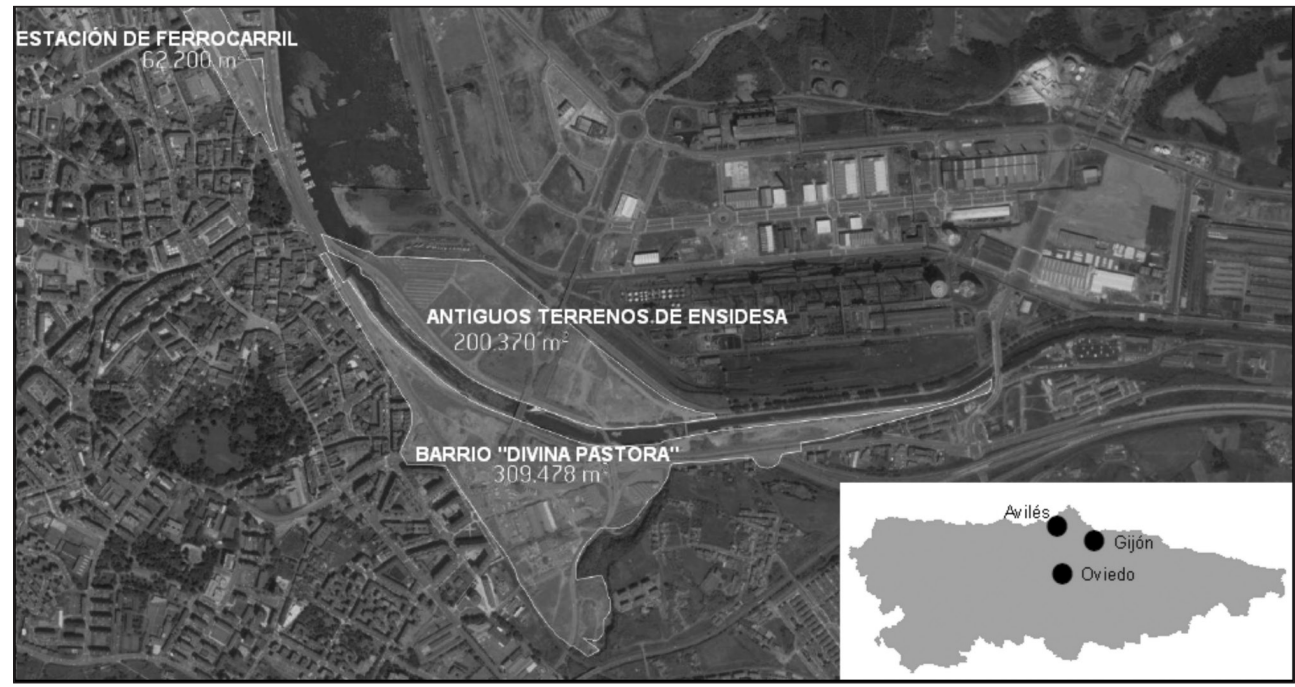

Fuente: MASTERPLAN Isla de la Innovación (www.isladelainnovacion.es)

\section{CONCLUSIONES}

A finales de la pasada década, como colofón a un libro sobre la historia de Avilés, De La Madrid (1999: 483-484) proponía un diagnóstico coherente con la situación de declive padecida en las dos últimas décadas del siglo XX, al afirmar: "Que el Avilés actual es un enfermo parece algo suficientemente probado, que la salida a su situación actual es incierta, sea para bien o para mal, también. Es decir, que si por algo se caracteriza esta ciudad es 
por la incertidumbre de su situación, en el momento en que se desmorona definitivamente un modelo económico y social al que la gran siderurgia había acostumbrado a esta comarca (...) La sociedad avilesina asiste apática a este proceso, sin haber tomado conciencia aún de su verdadera importancia y sin poner hasta ahora las primeras medidas que puedan mejorar su destino. No hay demasiadas iniciativas novedosas y las que aparecen nunca logran el consenso suficiente como para llegar a buen término».

Transcurridos poco más de diez años, las conclusiones que pueden deducirse de la investigación realizada son, en cambio, bastante distintas. En concreto, en la primera parte del trabajo hemos verificado cómo la dinámica demográfica comenzó a invertirse, gracias a unos saldos migratorios positivos que han permitido recuperar el crecimiento tras dos décadas de retroceso. Lo mismo cabría apuntar desde la perspectiva económica, donde a lo largo de la última década y hasta el inicio de la actual crisis se evidenció, no sólo la recuperación del empleo tras los duros ajustes impuestos por la reconversión industrial, sino también una evidente diversificación de la economía de la ciudad. Por otro lado, la multiplicación de actuaciones orientadas al desarrollo y, sobre todo, la sustitución de la apatía señalada por un creciente número de iniciativas locales, públicas y privadas, parece haber permitido la construcción de un nuevo proyecto de ciudad que cristaliza en la aprobación, no sin dificultades, del nuevo PGOU (2007). La evolución reciente de Avilés parece adecuarse, por tanto, a la idea de resilient city propuesta por algunos autores (Polèse, 2010), ante la capacidad demostrada de enfrentarse a impactos externos y adaptarse de forma positiva a un nuevo contexto.

Pero, pese a la puesta en marcha de un proceso de desarrollo que ha alcanzado ya resultados tangibles en diversos planos, la superación del declive exige un proceso largo, en el que aún son visibles algunas debilidades y retos que son fuente de incertidumbres respecto al futuro inmediato.

En relación con la diversificación económica y la nueva imagen urbana, la permanencia de las baterías de cok que Arcelor Mittal tiene alquiladas a la SEPI hasta 2017 (con posible prórroga hasta 2020) aún suscita ciertas controversias. Si bien en principio estaba prevista su desaparición en 1997, el aumento en el precio del cok y la escasez de este tipo de plantas en Europa hizo rentable el mantenimiento de una producción que supera anualmente el millón de toneladas, de la que se exporta más de la mitad y que se considera por algunos de valor estratégico para la región. Pero su contrapunto son unos impactos ambientales (polución, tráfico pesado) y visuales que algunos consideran difícilmente compatibles con su proximidad a los nuevos hitos urbanos como el Centro Cultural Niemeyer o la futura Isla de la Innovación, pese a la voluntad de convivencia mostrada por unos y otros.

La ya vieja polémica entre quienes apuestan por una ciudad postindustrial centrada en el turismo, la cultura y los servicios, frente a aquellos otros grupos e instituciones que valoran a la industria no tan sólo como parte del pasado, sino como uno de los pilares necesarios para su futuro, encuentra también su reflejo en los proyectos relativos al puerto. Para los primeros, un puerto destinado al tráfico de graneles y con grandes instalaciones industriales en sus orillas, algunas en deficiente estado de conservación, supone un freno para operaciones de renovación urbana como las llevadas a cabo en ciudades que han convertido esas áreas en espacios de oficinas, residenciales, culturales y de ocio, atrayendo importantes inversiones inmobiliarias. Para los segundos, el desplazamiento del tráfico pesado a la margen derecha de la ría puede permitir mayor compatibilidad de usos y mantener actividades (industriales, 
logísticas, pesqueras...) y empleos que siguen siendo necesarios, posibilitando una renovación pero no una metamorfosis urbana radical poco viable.

Otro reto es lograr una mejor inserción de Avilés en la sociedad del conocimiento. El esfuerzo realizado en esa dirección para apoyar la innovación tecnológica, mejorar el nivel formativo y, sobre todo, situar a la cultura en el centro de la actual estrategia de dinamización es indudable. Pero las herencias del pasado reciente también se resisten a desaparecer $\mathrm{y}$, en ese sentido, mientras buena parte de la I+D que realizan las grandes transnacionales se realiza fuera de la región, las pequeñas empresas aún generan una demanda insuficiente de innovaciones para los centros tecnológicos, al tiempo que su conexión con la universidad - apenas presente en la ciudad - sigue siendo débil. Finalmente, las características tradicionales del tejido empresarial local tampoco generan una oferta suficiente de empleos cualificados para buena parte de los jóvenes mejor formados, que emigran en bastantes casos a otras ciudades con mayores oportunidades laborales.

Esa competencia interurbana es, precisamente, otro aspecto a resaltar. Las ciudades próximas de Oviedo y Gijón, de mayor tamaño y dinamismo, pueden entenderse como un factor de reforzamiento de Avilés al ser parte de una aglomeración urbana polinuclear con una progresiva especialización de funciones. Pero también ejercen una evidente competencia por su mayor capacidad para atraer buena parte de los servicios y equipamientos de calidad o las sedes empresariales, localizar la residencia de los grupos profesionales más cualificados o disponer de mayor oferta hotelera. Al mismo tiempo, el puerto gijonés de El Musel, de mayor calado y que acaba de ser objeto de una nueva ampliación, cuenta con un tráfico de contenedores muy superior al de Avilés y todo ello se traduce en ventajas comparativas dentro de la nueva ordenación de la red portuaria en el seno de la Unión Europea.

En ese sentido, consolidar las redes de colaboración entre actores locales y externos que permitieron poner en marcha la mayor parte de proyectos aquí mencionados, o lograr una mejor articulación de la ciudad con su comarca, no sólo para la ordenación también para la planificación de su desarrollo, son otros dos retos importantes para que Avilés afronte en mejores condiciones la nueva crisis iniciada en 2008. Decisiones como la del Gobierno del Principado surgido de las elecciones autonómicas de 2011, que cerró el Centro Cultural Niemeyer en diciembre de ese año, apenas nueve meses después de su inauguración, pone en evidencia que los procesos de regeneración urbana son, por definición, dinámicos, por lo que su capacidad de adaptación a los cambios supone un reto permanente. A su vez, la defensa del Centro por múltiples organizaciones sociales y partidos políticos de la ciudad confirma la importancia de las redes locales, no sólo para construir procesos de desarrollo, sino también para enfrentarse al desmantelamiento de un proyecto valorado como estratégico y con evidente capacidad movilizadora.

\section{BIBLIOGRAFÍA}

AYUNTAMIENTO DE AVILÉS (2005): Documento de Revisión del Plan General de Ordenación Urbana de Avilés. Avilés (Asturias), Ayuntamiento de Avilés. Disponible en http:// www.ayto-aviles.es/html/planes_urban/PGOU-05_06/PGO.html 
AYUNTAMIENTO DE AVILÉS (2006): Diagnóstico de la economía de la ciudad de Avilés. Avilés, Ayuntamiento de Avilés y MER Consulting, Aviles. Disponible en http://www. aviles.es/AytoAvilesPortal/portal/cn/NavSec/Documento?IMAGEN=null

AYUNTAMIENTO DE AVILÉS (2008): Aviles Avanza, Ayuntamiento de Avilés. Disponible en http://www.aviles.es/pdf/AVILES\%20AVANZA.pdf?WT.mc_id=Aviles\%20 AVANZA.\%20Hacia\%20un\%20Territorio\%20Económica\%20y\%20Socialmente\%20 Responsable

BALLANCE, H. y SINCLAIR, S. W. (1983): Collapse and survival: industry strategies in a changing world. Londres. Allen\&Unwin.

BENITO, P. (1992): El espacio industrial en Asturias. Barcelona. Oikos-Tau.

BENITO, P. (2004): «Discursos, propuestas y acciones sobre la ciudad postindustrial». Anales de Geografía de la Universidad Complutense, n 29, 9-29.

BENITO, P. y LÓPEZ, A. (2008): «Patrimonio industrial y nuevas perspectivas funcionales para las ciudades en reestructuración». Estudios Geográficos, n 264, 23-50.

BIRCH, K., MACKINNON, D. y CUMBERS, A. (2010): «Old industrial regions in Europe: a comparative assessment of economic performance». Regional Studies, $\mathrm{n}^{\circ} 44$ (1), 35-53.

CAMAGNI, R. y MAILLAT, D. (Eds.) (2006): Milieux innovateurs. Théorie et politiques. París. Economica.

CARAVACA, I. y GONZÁLEZ, G. (2009): «Las redes de colaboración como base del desarrollo territorial» en Scripta Nova, vol. XIII, núm. 289, 1 de mayo de 2009. Universidad de Barcelona. Disponible en http://www.ub.edu/geocrit/sn/sn-289.htm

CHESHIRE, P. y HAY, D. (1989): Urban problems in Western Europe. Londres. Unwin Hyman.

DE LA MADRID, J. C. (1999): Avilés, una historia de mil años. Gijón, Azucel.

ESTEBAN, M. (2000): Bilbao, luces y sombras del titanio. El proceso de regeneración del Bilbao metropolitano. Bilbao. Universidad del País Vasco.

FLORIDA, R. (2002): The rise of the creative class: and how it's transforming work, leisure, community, and everyday life. Nueva York. Basic Books.

FOL, S. y CUNNINGHAM-SABOT, E. (2010): «Déclin urbain et shrinking cities: une evaluation critique des approches de la décroissance urbaine». Annales de Géographie, ${ }^{\circ}$ 674, 359-383.

FONTAN, J. M., KLEIN, J. L. y TREMBLAY D. G. (2005): Innovation socioterritoriales et reconversion économique: le cas de Montréal. París. L'Harmattan.

FOTHERGILL, S., KITSON, M. y MONK, S. (1988): Urban industrial decline: the causes of urban/rural contrasts in manufacturing employment change. Londres. HMSO.

GONZÁLEZ, S. (2006): «Scalar narratives in Bilbao. A cultural politics of scales approach to the study of urban policy». International Journal of Urban and Regional Research, $\mathrm{n}^{\circ}$ 30 (4), 836-857.

HALBERT, L. (2005): «Le desserrement intra-métropolitain des emplois d'intermediation: une tentative de mesure el d'interpretation dans le cas de la región métropolitaine parisienne». Géographie, Économie, Société, no 7 (1), 1-20.

HEALEY, P. (1997): Collaborative planning: shaping places in fragmented societies, Vancouver. UBC Press.

KOOIMAN, J. (2003): Governing as governance. Londres. Sage. 
LANDABASO, M. y DÍEZ, M. A. (1989): Regiones europeas de antigua industrialización. Propuestas frente al reto tecnológico. Bilbao. SPRI.

LEIRA, E. y CALVO, L. (2003): «Avilés ante un nuevo futuro». Ciudades: Revista del Instituto Universitario de Urbanística de la Universidad de Valladolid, $\mathrm{n}^{\mathrm{o}}$ 7, 103-128.

MARTIN, R. y SUNLEY, P. (2006): «Path dependence and regional economic evolution». Journal of Economic Geography, n 6, 395-437.

MÉNDEZ, R. (2002): «Innovación y desarrollo territorial: algunos debates teóricos recientes». Revista EURE, vol. XXVIII, n ${ }^{\circ}$ 84, 63-83.

MÉNDEZ, R. (Ed.) (2010): Estrategias de innovación industrial y desarrollo económico en las ciudades intermedias de España. Madrid. Fundación BBVA.

MÉNDEZ, R. y SÁNCHEZ MORAL, S. (2010): «Spanish cities in the knowledge economy: theoretical debates and empirical evidence». European Urban and Regional Studies, vol. $18, \mathrm{n}^{\circ} 2,136-155$.

MORALES, G. (1982): Industria y espacio urbano en Avilés, 2 vols. Gijón. Silverio Cañada.

MUSTERD, S. et al. (2007): Accomodating creative knowledge. A literature review from a European perspective. ACRE Report 1. Amsterdam. AMIDSt, University of Amsterdam.

PASCUAL, J. M. y GODÀS, X. (Coord.) (2010): El buen gobierno 2.0: la gobernanza democrática territorial. Valencia. Tirant lo Blanch.

PIKE, A., DAWLEY, S. y TOMANEY, J. (2010): «Resilience, adaptation and adaptability». Cambridge Journal of Regions, Economy and Society, $\mathrm{n}^{\circ}$ 3, 59-70.

POLÈSE, M. (2010): «The resilient city: on the determinants of successful urban economies» en Working Paper $n^{\circ}$ 2010-03, Montreal. University of Quebec, Centre-Urbanisation Culture Société. INRS.

POWER, A., PLÖGER, J. y WINKER, A. (2010): Phoenix cities: The fall and rise of great industrial cities. Bristol. Policy Press.

PRADA, J. (2011): Desarrollo, patrimonio y políticas de revitalización en ciudades intermedias de especialización minero-industrial. El caso de Langreo (Asturias). Oviedo. Consejo Económico y Social de Asturias.

RHODES, J. (1986): «Regional dimensions of industrial decline» en The Geography of DeIndustrialisation (Martin, R. y Rowthorn, B., Eds.). Londres. Macmillan, 138-168.

RODRÍGUEZ, F., MENÉNDEZ, R. y BLANCO, J. (2009): El área metropolitana de Asturias. Ciudad Astur: el nacimiento de una estrella urbana en Europa. Gijón. Trea.

SALOM, J. y ALBERTOS, J. M. (Eds.) (2009): Redes socioinstitucionales, estrategias de innovación y desarrollo territorial en España. Valencia. Publicaciones Universidad de Valencia.

SÁNCHEZ MORAL, S.; PRADA, J. y MÉNDEZ, R. (2009): «Dinámicas de las ciudades de tamaño intermedio en el sistema urbano español: entre el declive y la recuperación». En Actas del XXI Congreso de Geógrafos Españoles, Ciudad Real 27-29 de octubre de 2009, pp. 655-670.

SCOTT, A. J. (2006): «Creative cities: conceptual issues and policy questions». Journal of Urban Affairs, $\mathrm{n}^{\circ} 28$ (1), 1-17.

SIMMIE, J. y CARPENTER, J. (2008): Path dependence and the evolution of city regional economies. Londres. NESTA. 
SUNLEY, P. (2008): «Relational economic geography: a partial understanding or a new paradigm?». Economic Geography, n 84, 1-26.

TUROK, I. y MYKHNENKO, V. (2007): «The trajectory of European cities, 1960-2005». Cities, $\mathrm{n}^{\circ} 24$ (3), 165-182.

TUROK, I. y MYKHNENKO, V. (2008): «Resurgent European cities?». Urban Research\& Practice, $\mathrm{n}^{\mathrm{o}} 1$ (1), 54-77.

VAN DEN BERG, L. et al. (1982): Urban Europe: a study of growth and decline. Oxford. Pergamon Press.

VAN WINDEN, W., VAN DEN BERG, L. y POL, P. (2007): «European cities in the knowledge economy: towards a typology». Urban Studies, nº 44 (3), 525-549. 
